\title{
Lateral retropharyngeal lymph node metastasis from squamous cell carcinoma of the upper gingiva: A case report
}

\author{
HIROSHI YAMAZAKI $^{1,2}$, MASASHI SASAKI $^{1}$, KEN-ICHI AOYAMA $^{1,2}$, TAKATSUGU SUZUKI $^{1}$, YUYA DENDA ${ }^{1}$, \\ MASAHIRO UCHIBORI ${ }^{1}$, YASUHIRO NAKANISHI ${ }^{1}$, RENA KOJIMA $^{1,2}$ and YOSHIHIDE OTA ${ }^{1}$ \\ ${ }^{1}$ Department of Oral and Maxillofacial Surgery, Tokai University School of Medicine, Isehara, Kanagawa 259-1193; \\ ${ }^{2}$ Department of Oral and Maxillofacial Surgery, Tokai University Oiso Hospital, Oiso, Kanagawa 259-0198, Japan
}

Received August 1, 2017; Accepted November 9, 2017

DOI: $10.3892 / \mathrm{mco} .2017 .1496$

\begin{abstract}
Lateral retropharyngeal lymph node (LRPLN) is located between the internal carotid artery and the prevertebral muscles. Metastasis to the LRPLN is frequent in nasopharyngeal cancer, but is rare in oral cancer. The prognosis of patients with oral cancer with LRPLN metastasis is usually poor. The present study reported a patient with LRPLN metastasis from squamous cell carcinoma of the upper gingiva, who survived for $>7$ years. Docetaxel, cisplatin and fluorouracil (TPF) therapy was performed as induction chemotherapy and it was planned to subsequently conduct chemoradiotherapy or surgery. As the tumor only exhibited a transient response to TPF, surgery was selected. Postoperatively, only radiotherapy was performed and a favorable outcome was achieved.
\end{abstract}

\section{Introduction}

The lateral retropharyngeal lymph node (LRPLN) is located between the internal carotid artery and the prevertebral muscles. The LRPLN is most often seen anterior to the arch of $\mathrm{C} 1$, but is sometimes found at the level of the soft palate. The uppermost part of the LRPLN anterior to the atlas is known as the lymph node of Rouvière (1). Lateral retropharyngeal lymph node (LRPLN) metastasis from oral squamous cell carcinoma (OSCC) is rare and the prognosis is extremely poor (2-5). We report an unusual patient with LRPLN metastasis from squamous cell carcinoma of the upper gingiva and no progression for more than 7 years. Docetaxel, cisplatin and fluorouracil (TPF) therapy $(6,7)$ was performed as induction chemotherapy and it was planned to subsequently conduct chemoradiotherapy or surgery. As the tumor only exhibited a transient response to

Correspondence to: Dr Hiroshi Yamazaki, Department of Oral and Maxillofacial Surgery, Tokai University Oiso Hospital, 21-1 Gakkyo, Oiso, Kanagawa 259-0198, Japan

E-mail: osyamaza@is.icc.u-tokai.ac.jp

Key words: lateral retropharyngeal lymph node, metastasis, oral cancer, induction chemotherapy
TPF, surgery was selected. Postoperatively, only radiotherapy was performed and a favorable outcome was achieved.

\section{Case report}

A 56-year-old Japanese woman visited the outpatient clinic of the Department of Oral Surgery at Tokai University Hospital in 2009. She had a gingival ulcer near the right maxillary second molar. This molar had been extracted at another clinic 2 months previously, but healing of the socket was poor. A mobile lymph node measuring $1.5 \mathrm{~cm}$ was palpable in the right cervical region (Fig. 1). Her medical history included fatty liver with hepatic impairment and her performance status (ECOG) was Grade 0. A panoramic X-ray revealed bone destruction with an uneven margin of the right maxillary molar socket. Contrast CT scans showed a tumor with heterogeneous enhancement that spread from the right maxillary region through the maxillary sinus, and bone destruction with an uneven margin was observed. Enlarged lymph nodes with rim enhancement were seen in the right submandibular, superior internal jugular vein, and LRPLN regions (Fig. 2). PET/CT also demonstrated abnormal accumulation at the same sites. The SUVmax of the primary tumor was 17.2. Biopsy revealed squamous cell carcinoma and the diagnosis was upper gingival carcinoma T4aN2bM0: Stage IV A (Fig. 3).

Laboratory tests gave the following results: WBC $8.6 \times 10^{3} / \mu 1$ (Seg+Stab $73.5 \%$, lymphocytes $20.4 \%$, monocytes $5.1 \%$, eosinophils $0.8 \%$, basophils $0.2 \%$ ), RBC $3.93 \times 10^{6} / \mu 1, \mathrm{Hb}$ $12.7 \mathrm{~g} / \mathrm{dl}$, Ht 37.3\%, Plt 32.3×10 $/ \mu 1$, AST $34 \mathrm{U} / 1$, ALT $40 \mathrm{U} / 1$, LDH 202 U/1, ALP 244 U/l, $\gamma$-GTP 62 U/1, BUN 13 mg/dl, Cre $0.6 \mathrm{mg} / \mathrm{dl}$, TP $8.1 \mathrm{~g} / \mathrm{dl}$, Glu $103 \mathrm{mg} / \mathrm{dl}$, TG $84 \mathrm{mg} / \mathrm{dl}$, T-CHO $193 \mathrm{mg} / \mathrm{dl}, \mathrm{Na} 141 \mathrm{mEq} / 1, \mathrm{~K} 4.1 \mathrm{mEq} / 1, \mathrm{Cl} 107 \mathrm{mEq} / 1$, and T-bil $0.4 \mathrm{mg} / \mathrm{dl}$. Mild hepatic impairment was observed.

Three courses of the induction chemotherapy were conducted with docetaxel (Taxotere, Sanofi-Aventis), cisplatin (CDDP), and fluorouracil (TPF) therapy (docetaxel at $60 \mathrm{mg} / \mathrm{m}^{2}$, CDDP at $60 \mathrm{mg} / \mathrm{m}^{2}$, and fluorouracil at $\left.600 \mathrm{mg} / \mathrm{m}^{2}\right)(6,7)$. At the end of the first course, CT demonstrated reduction in the size of the primary lesion and lymph nodes. However, repeat CT after 3 courses showed re-enlargement of metastatic lymph nodes, although the primary tumor was unchanged. There was only one adverse event of Grade 3 or higher during chemotherapy, which was leukopenia. 
Thus, the primary tumor and LRPLN responded to the first course, but regrowth of the LRPLN was observed after 3 courses (Fig. 4). Similarly, the right submandibular lymph node initially decreased in size, but then showed regrowth. Therefore, she was judged to have progressive disease and it was decided to perform surgery. After induction of general anesthesia, surgery was started with tracheotomy followed by bilateral modified radical neck dissection. A midline lower lip incision was made and the mandibular swing approach was employed. The right maxilla was resected. From the dorsal region of the submandibular gland, fatty tissue was removed from the parapharyngeal space and it was explored in the cranial direction until the base of the skull was observed The fascia of the superior constrictor muscle was detached. The internal carotid artery and vagus nerve were exposed, and were pulled aside using vascular tapes. Then the internal carotid artery, vagus nerve, and sympathetic nerves were dissected up to the base of the skull and fatty tissue was removed from the prevertebral fascia to complete dissection of the retropharyngeal space (Figs. 5 and 6). Postoperatively, only radiotherapy was administered (60 Gy). At 7 years after surgery, there has been no sign of relapse or metastasis. The patient has no difficulty with eating, swallowing, or speaking, and she returned to work postoperatively.

Histopathological examination of resected specimens from the primary lesion indicated that the sites where tumor cells presumably had been present were replaced by fibrous tissue showing mild edema. Inflammatory cells (mainly lymphocytes) were observed at these sites, but no tumor cells were seen. Some LRPLN sections showed fibrosis and cyst formation within the structure of the lymph node, but proliferation of cancer cells was also observed and part of the capsule was involved. Tumor cells with enlarged, densely stained nuclei were observed, suggesting changes induced by chemotherapy (Fig. 7).

The present study was approved by the Institutional Review Board for Clinical Research of Tokai University (Kanagawa, Japan). Written informed consent was obtained from the patient for publication of this case report and any accompanying images.

\section{Discussion}

The LRPLN is located between the internal carotid artery and the prevertebral muscles. The LRPLN is most often seen anterior to the arch of $\mathrm{C} 1$, but is sometimes found at the level of the soft palate. The uppermost part of the LRPLN anterior to the atlas is known as the lymph node of Rouvière (1). The incidence of LRPLN metastasis is reported to be 29.1-88.6\% in patients with nasopharyngeal cancer, $16-50 \%$ in those with oropharyngeal cancer, and 6-20\% in those with hypopharyngeal cancer (1). The incidence is reported to be $0.6-1.4 \%$ in patients with OSCC $(3,5)$, while it is $6.9-16 \%$ in patients with maxillary cancer and upper gingival cancer $(4,8)$. Thus, compared with oral cancer at other sites, the incidence of LRPLN metastasis is high among patients with upper gingival cancer. At our institution, LRPLN metastasis was detected in 3 out of 57 patients with upper gingival cancer (5.2\%) between 2003 and 2013. Umeda et al (4) discussed the route of LRPLN metastasis in patients with upper gingival cancer and they concluded that, similar to oral cancer at other sites, it occurs via the submandibular nodes and superior internal jugular nodes in upper gingival

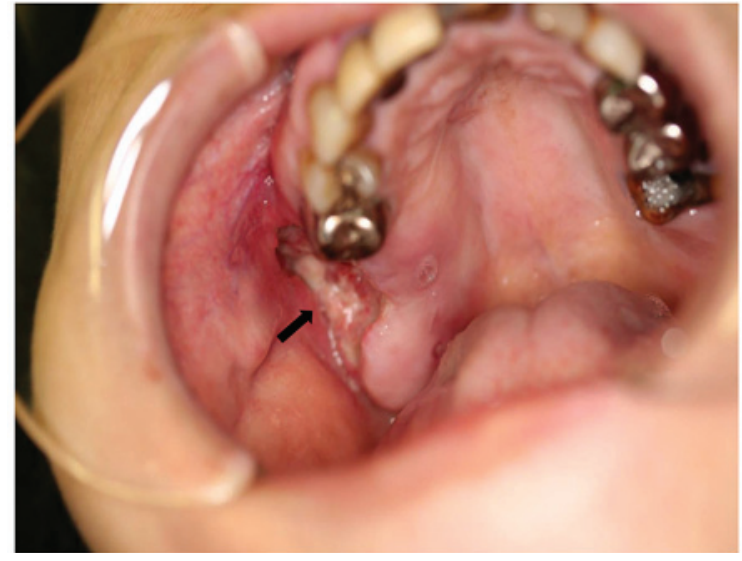

Figure 1. Oral findings at the initial examination: There is an ulcer adjacent to the right upper molar region.

cancer (4). Lymph channels inside the maxilla also enter both of these lymph nodes. Furthermore, there may be a route unique to maxillary cancer that reaches the LRPLN from tumors adjacent to the anterior and posterior teeth, although it is more developed for the posterior teeth, which means that tumors growing posteriorly tend to metastasize to the LRPLN. Moreover, metastasis to the superior internal jugular lymph nodes could subsequently result in retrograde metastasis to the LRPLN. Therefore, it is considered that LRPLN metastasis from upper gingival cancer may be different to metastasis from other oral cancers.

In many reports about LRPLN metastasis from upper gingival cancer (2-5), it is stated that metastasis to this node occurred following resection of the primary tumor or secondary lymph node metastases and it is common for there to be multiple metastases to other lymph nodes (9). Accordingly, it seems that retrograde metastasis may often occur in patients with secondary lymph node metastasis.

For treatment of LRPLN metastasis, surgery is often considered in patients with hypopharyngeal cancer $(10,11)$. Elective neck dissection and adjuvant radiotherapy are recommended. LRPLN metastasis tends to progress rapidly to involve the carotid sheath. Accordingly, the prognosis is usually quite poor when LRPLN metastasis is detected (2-4). Because there are not so many patients with oral cancer, including upper gingival cancer, evaluation of treatment outcomes has rarely been conducted.

When a patient first presents with a tumor and LRPLN metastasis, curative treatment is attempted with chemoradiotherapy (CRT), radiotherapy alone, neo-adjuvant chemotherapy + surgery, or CRT + surgery + adjuvant CRT (5). Alternatively, chemotherapy is given alone as palliative therapy. Patients with secondary metastasis are often treated by radiotherapy alone or chemotherapy alone (5). LRPLN metastasis can only be detected by CT or MRI and many tumors are already non-resectable when detected, which means that radiotherapy or chemotherapy must be chosen.

On the other hand, there is a small group of patients in whom surgery is effective $(3,4)$. Upper gingival cancer that grows posteriorly with metastasis in the deep cervical area is considered a high-risk tumor for LRPLN metastasis. Dissection of the parapharyngeal space and retropharyngeal space should be conducted and resection of the entire lesion 

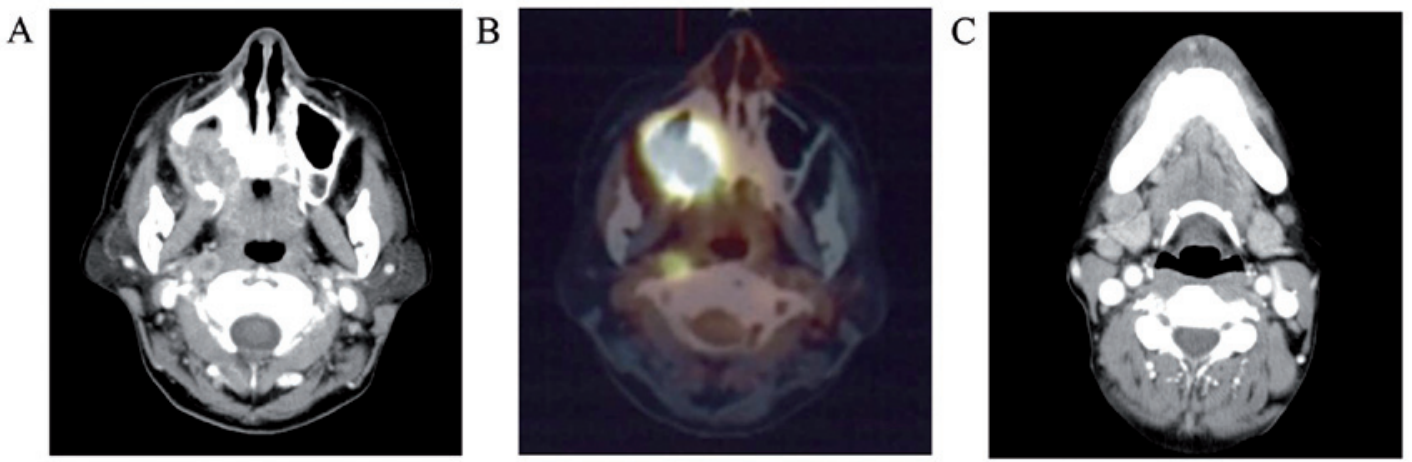

Figure 2. (A) Contrast CT (initial scan): The posterior part of the right maxilla is destroyed by tumor. The right LRPLN is enlarged and shows rim enhancement. (B) PET-CT finding: There is abnormal accumulation in the posterior part of the right maxilla and in the right LRPLN. (C) Contrast CT (initial scan): Metastasis to the right submandibular lymph nodes is observed. LRPLN, lateral retropharyngeal lymph node.

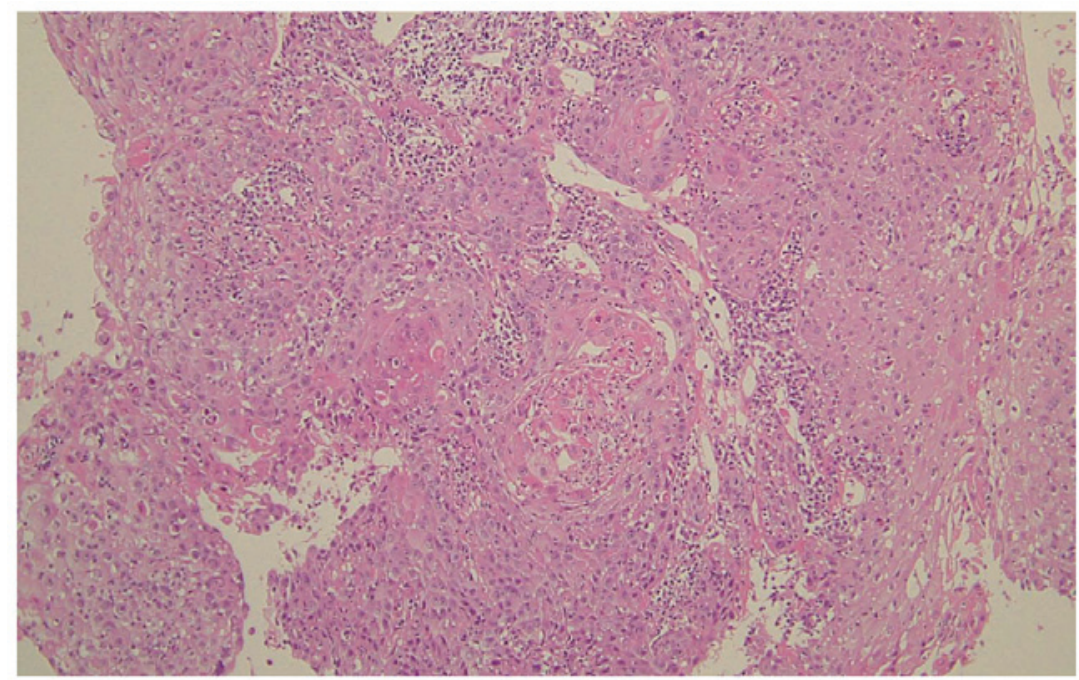

Figure 3. Biopsy specimen (H\&E staining). Well-differentiated squamous cell carcinoma. Magnification, x100. H\&E, haematoxylin and eosin.
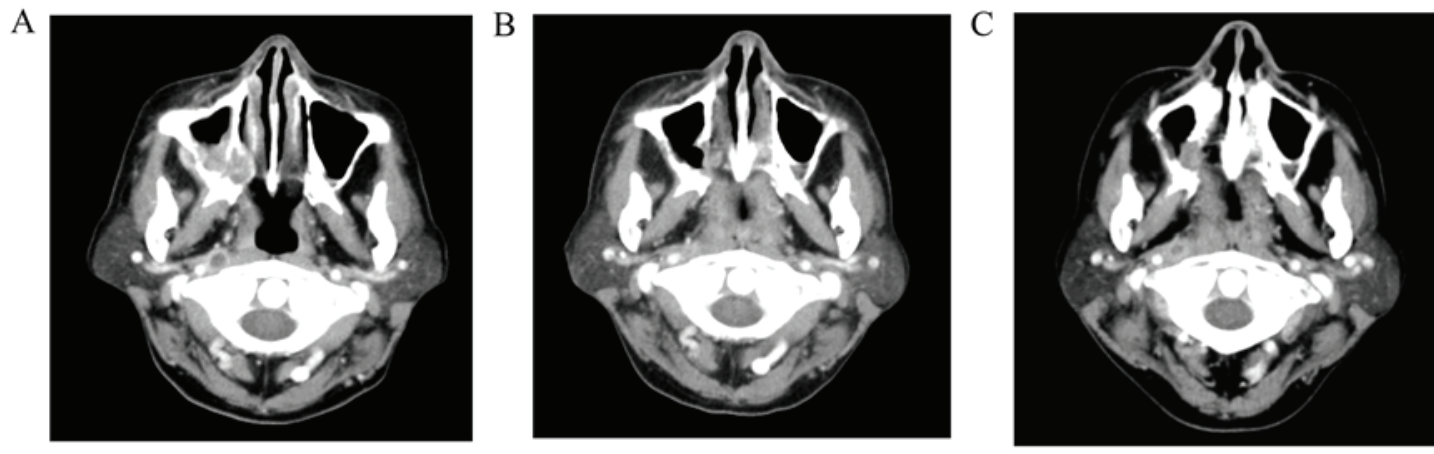

Figure 4. Contrast CT (before and after chemotherapy). (A) At the initial examination, (B) after 1 course of chemotherapy, there was a reduction in tumor size and (C) After 3 courses of chemotherapy, there was tumor regrowth.

together with the primary tumor should be considered $(4,12)$. There have been no reports of a favorable outcome with current standard therapy or CRT according to the National Comprehensive Cancer Network (NCCN) strategy, in which surgery is followed by high-dose CDDP $\left(100 \mathrm{mg} / \mathrm{m}^{2}\right.$ on days 1, 22, and 43) (13). When LRPLN metastasis occurs, it may be debatable whether resection with a sufficient margin is feasible.
In the present case, induction chemotherapy was provided and it was planned to subsequently conduct CRT or surgery (cetuximab was not available in Japan in 2009). However, surgery was selected as the tumor only transiently responded to TPF therapy. Since TPF therapy had been conducted prior to surgery, postoperative radiotherapy was performed alone to improve tolerability and a favorable outcome was achieved. It is debatable whether our patient should be judged as resectable 
A

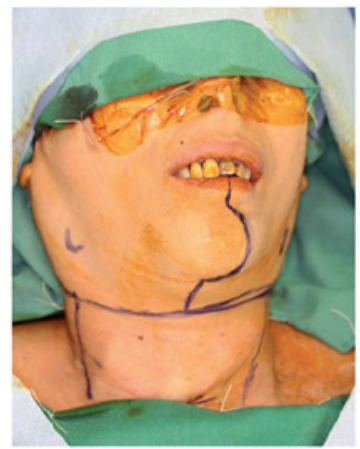

B

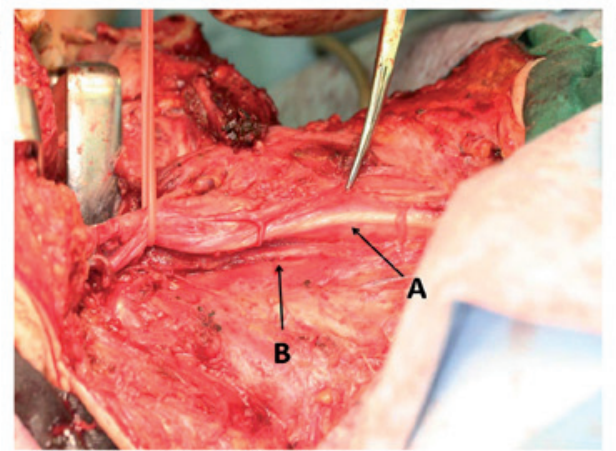

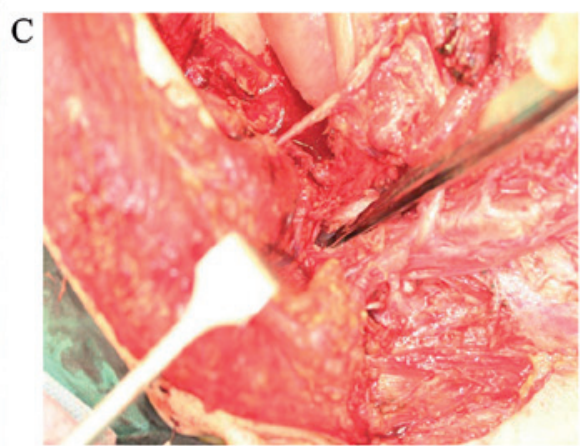

Figure 5. Surgical findings. (A) Intraoperative photograph (skin resection line). Partial resection of the right maxilla, bilateral modified radical neck dissection, right LRPLN dissection and reconstruction with a free skin flap were conducted. (B) Intraoperative photograph (right neck). The carotid sheath (common carotid artery and vagus nerve) (arrow A) and sympathetic nerves (arrow B) are demonstrated. (C) Intraoperative photograph (right neck). LRPLN metastasis is seen (at the forceps tip). LRPLN, lateral retropharyngeal lymph node.

A

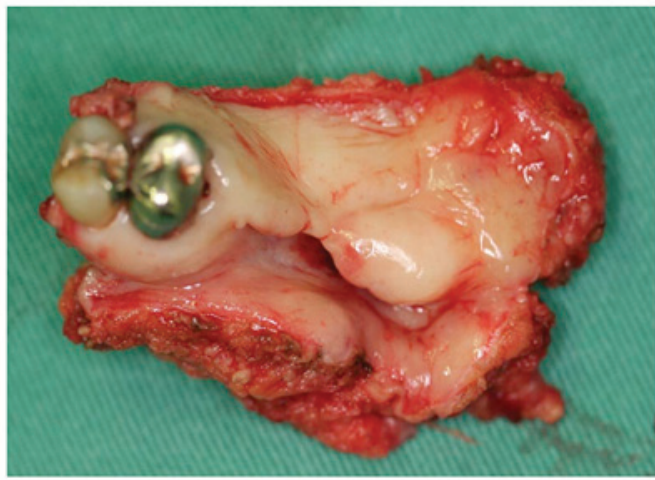

B

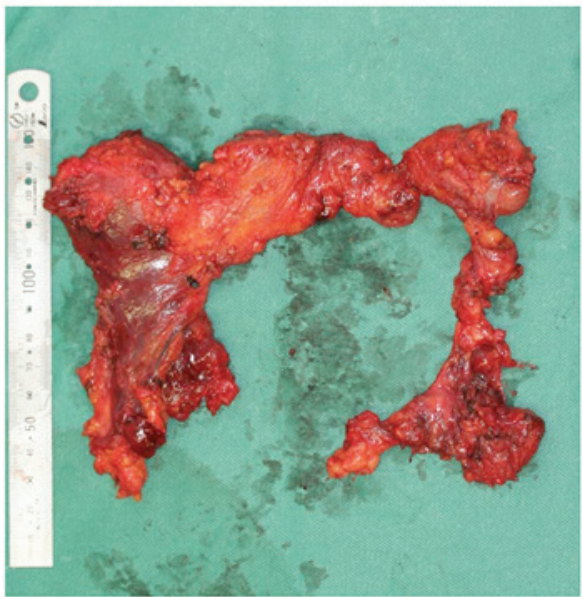

Figure 6. Resected specimens. (A) Resected maxillary tissue. (B) Dissected neck tissue.
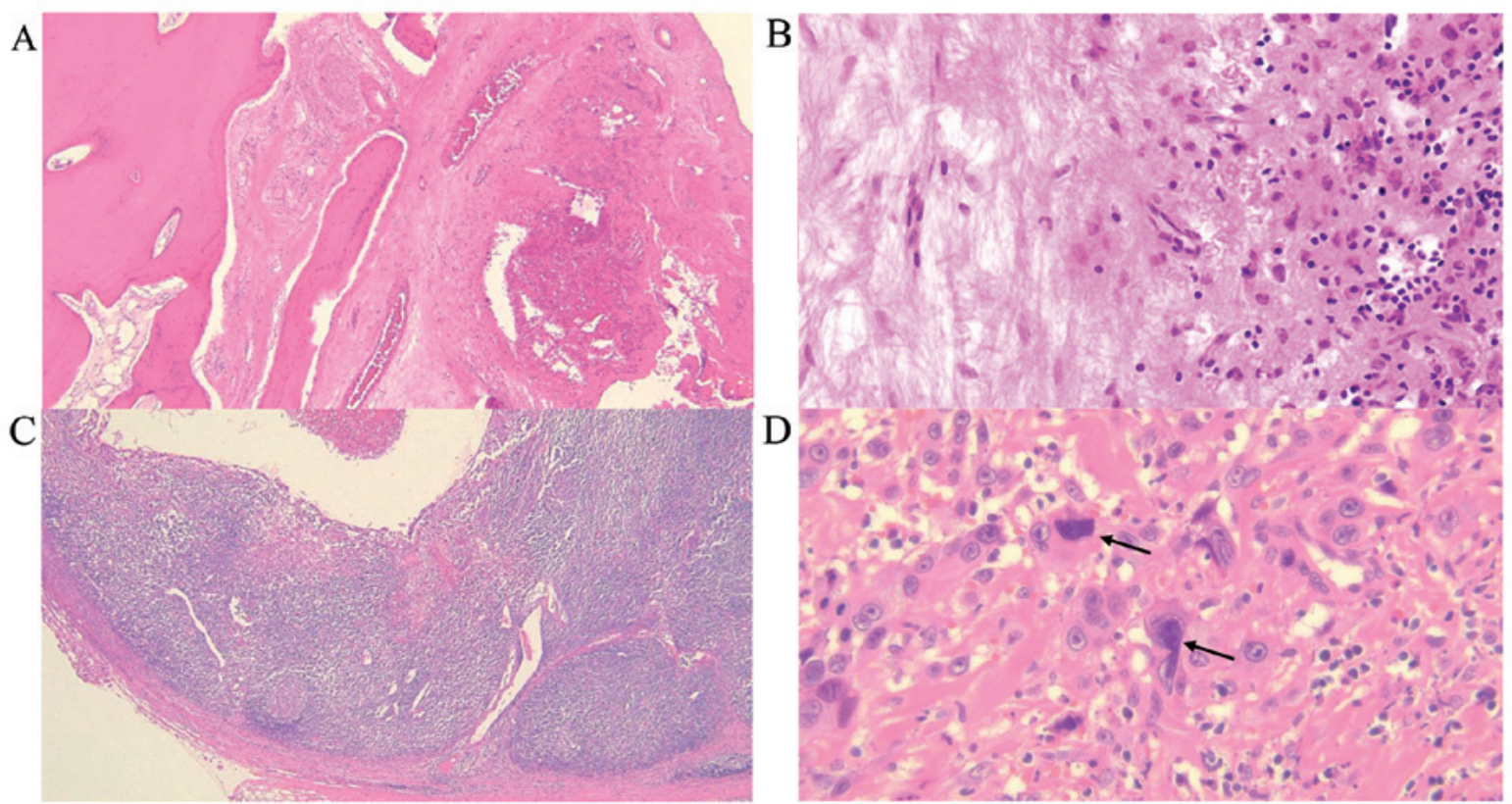

Figure 7. Histopathological findings (H\&E staining). (A) A section of the primary tumor (magnification, $\mathrm{x} 40$ ) shows fibrosis between bone tissues where the tumor may have been present. (B) Primary tumor (magnification, x200). Inflammatory cells (mainly lymphocytes) were observed at these sites, but no tumor cells were seen. (C) LRPLN (magnification, x40). Although fibrosis and cysts are present within the lymph node, proliferation of cancer cells is also observed. Part of the capsule is involved by the tumor. (D) LRPLN (magnification, x400). Tumor cells with enlarged, densely stained nuclei were observed, suggesting changes induced by chemotherapy (arrow). H\&E, haematoxylin and eosin; LRPLN, lateral retropharyngeal lymph node. 
or non-resectable. Induction chemotherapy was reported to be ineffective for resectable OSCC $(14,15)$. Standard therapy for non-resectable OSCC is CRT with high-dose CDDP (13), while induction chemotherapy with TPF therapy is also regarded as standard therapy in Europe (16). Among the regimens for induction chemotherapy, TPF therapy is considered to be the standard (17). On the other hand, a prospective Phase III study and a meta-analysis both failed to show an additive effect of induction chemotherapy (18-20), so re-appraisal of TPF therapy may be needed.

In conclusion, further discussion about whether treatment of LRPLN metastasis was appropriate in the present case seems to be warranted. Since LRPLN metastasis is rare among patients with oral cancer, a multicenter study will be needed to accumulate more cases.

\section{References}

1. Coskun HH, Ferlito A, Medina JE, Robbins KT, Rodrigo JP, Strojan P, Suárez C, Takes RP, Woolgar JA, Shaha AR, et al: Retropharyngeal lymph node metastases in head and neck malignancies. Head Neck 33: 1520-1529, 2011.

2. Kimura Y, Hanazawa T, Sano T and Okano T: Lateral retropharyngeal node metastasis from carcinoma of the upper gingiva and maxillary sinus. AJNR Am J Neuroradiol 19: 1221-1224, 1998.

3. Nishida M, Yasuda S, Murakami K, Yamamura I, Nagata Y and Iizuka T: Retropharyngeal lymph node metastases from oral cancer: A report of 2 patients. J Oral Maxillofac Surg 63: 410-412, 2005.

4. Umeda M, Shigeta T, Takahashi H, Kataoka T, Oguni A, Minamikawa T, Shibuya Y, Yokoo S and Komori T: Metastasis to the lateral retropharyngeal lymph node from squamous cell carcinoma of the oral cavity: Report of three cases. Int J Oral Maxillofac Surg 38: 1004-1008, 2009.

5. Tseng JR, Ho TY, Lin CY, Lee LY, Wang HM, Liao CT and Yen TC: Clinical outcomes of patients with oral cavity squamous cell carcinoma and retropharyngeal lymph node metastasis identified by FDG PET/CT. PLoS One 8: e79766, 2013.

6. Vermorken JB, Remenar E, van Herpen C, Gorlia T, Mesia R, Degardin M, Stewart JS, Jelic S, Betka J, Preiss JH, et al: Cisplatin, fluorouracil and docetaxel in unresectable head and neck cancer. N Engl J Med 357: 1695-1704, 2007.

7. Posner MR, Hershock DM, Blajman CR, Mickiewicz E, Winquist E, Gorbounova V, Tjulandin S, Shin DM, Cullen K, Ervin TJ, et al: Cisplatin and fluorouracil alone or with docetaxel in head and neck cancer. N Engl J Med 357: 1705-1715, 2007.

8. Watarai J, Seino Y, Kobayashi M, Shindo M and Kato T: CT of retropharyngeal lymph node metastasis from maxillary carcinoma. Acta Radiol 34: 492-495, 1993.

9. Tauzin M, Rabalais A, Hagan JL, Wood CG, Ferris RL and Walvekar RR: PET-CT staging of the neck in cancers of the oropharynx: Patterns of regional and retropharyngeal nodal metastasis. World J Surg Oncol 8: 70, 2010.
10. Amatsu M, Mohri M and Kinishi M: Significance of retropharyngeal node dissection at radical surgery for carcinoma of the hypopharynx and cervical esophagus. Laryngoscope 111: 1099-1103, 2001

11. Kamiyama R, Saikawa M and Kishimoto S: Significance of retropharyngeal lymph node dissection in hypopharyngeal cancer. Jpn J Clin Oncol 39: 632-637, 2009.

12. Umeda M, Minamikawa T, Komatsubara H, Ojima Y, Shibuya Y, Yokoo S and Komori T: En bloc resection of the primary tumour and cervical lymph nodes through the parapharyngeal space in patients with squamous cell carcinoma of the maxilla: A preliminary study. Br J Oral Maxillofac Surg 43: 17-22, 2005.

13. Pfister DG, Spencer S, Brizel DM, Burtness B, Busse PM, Caudell JJ, Cmelak AJ, Colevas AD, Dunphy F, Eisele DW, et al: Head and neck cancers, Version 2.2014. Clinical practice guidelines in oncology. J Natl Compr Canc Netw 12: 1454-1487, 2014.

14. Marta GN, Riera R, Bossi P, Zhong LP, Licitra L, Macedo CR, de Castro Junior G, Carvalho AL, William WN Jr and Kowalski LP: Induction chemotherapy prior to surgery with or without postoperative radiotherapy for oral cavity cancer patients: Systematic review and meta-analysis. Eur J Cancer 51: 2596-2603, 2015.

15. Lau A, Li KY, Yang WF and Su YX: Induction chemotherapy for squamous cell carcinomas of the oral cavity: A cumulative meta-analysis. Oral Oncol 61: 104-114, 2016.

16. Grégoire V, Lefebvre JL, Licitra L and Felip E: EHNS-ESMO-ESTRO Guidelines Working Group: Squamous cell carcinoma of the head and neck: EHNS-ESMO-ESTRO Clinical Practice Guidelines for diagnosis, treatment and follow-up. Ann Oncol 21 (Suppl 5): v184-v186, 2010.

17. Teo M, Karakaya E, Young CA, Dyker KE, Coyle C, Sen M and Prestwich RJ: The efficacy of induction chemotherapy with docetaxel, cisplatin and 5-fluorouracil combined with cisplatin concurrent chemoradiotherapy for locally advanced head and neck squamous cell carcinoma: A matched pair analysis. Clin Oncol (R Coll Radiol) 25: 647-653, 2013.

18. Zhang L, Jiang N, Shi Y, Li S, Wang P and Zhao Y: Induction chemotherapy with concurrent chemoradiotherapy versus concurrent chemoradiotherapy for locally advanced squamous cell carcinoma of head and neck: A meta-analysis. Sci Rep 5: 10798, 2015.

19. Hitt R, Grau JJ, López-Pousa A, Berrocal A, García-Girón C, Irigoyen A, Sastre J, Martínez-Trufero J, Brandariz Castelo JA, Verger E, et al: A randomized phase III trial comparing induction chemotherapy followed by chemoradiotherapy versus chemoradiotherapy alone as treatment of unresectable head and neck cancer. Ann Oncol 25: 216-225, 2014

20. Budach W, Bölke E, Kammers K, Gerber PA, Orth K, Gripp S and Matuschek C: Induction chemotherapy followed by concurrent radio-chemotherapy versus concurrent radio-chemotherapy alone as treatment of locally advanced squamous cell carcinoma of the head and neck (HNSCC): A meta-analysis of randomized trials. Radiother Oncol 118: 238-243, 2016. International (CC BY-NC-ND 4.0) License. 